\title{
MEMO and paediatric oncology - Yes, we can!
}

\begin{abstract}
MEMO - the Magazine of European Medical Oncology - is flourishing and stepping into its second year, aiming at providing oncology professionals with news and information relevant to their daily practice. Hence, time has ripened to integrate also paediatric oncology and haematology in its scope. From now on, the adult and the paediatric field will therefore join forces in MEMO and try to elicit mutual stimulation, interest and interaction for the sake of our patients.

Many new developments in oncology, such as the recent implementation of signal intercepting therapies, have spilled over from the adult setting to the paediatric field. However, the peculiar and successful ways of practice in paediatric oncology are increasingly influencing also adult care, specifically when it comes to treat young adults with cancers typical for childhood and adolescence. To this end, stimulating and exemplary insights into the biology of certain cancers have been made in particular by paediatric research. MEMO will be dedicated to highlight recent and new developments at the intersection of paediatric and adult oncology, and where paediatric oncology is at the forefront of research.

Paediatric oncology has made extraordinary progress within the past 30 years. Since childhood/adolescence cancers are relatively rare diseases (Thanks be to God!) much of the achievements were made on the basis of international co-operations, both in laboratory and in clinical research. The cornerstone of such co-operations has been paediatric treatment optimization studies, in which there is a long tradition throughout the European countries. With the new organizational and legal framework of the EU, also the way of co-operating in paediatric oncology in Europe is about to change and there is a need for adaptations. To promote our issues in the new era we need to speak with "one voice" - to this end, a platform for communication and information as well as a sounding board for European paediatric oncology clinicians is needed. MEMO will serve also these issues in the future. As a first step, this year one of the up-coming issues of MEMO will be dedicated to highlight the current problems and needs of paediatric oncology in Europe.

Central Europe is the gateway of MEMO - definitely also for the paediatric section. Leading paediatric oncology representatives from several countries in the region have now joined the editorial board of MEMO and the list will soon expand with representatives from other European countries. Hence, MEMO is devoted to publish paediatric oncology contributions specifically from central European countries to promote mutual perception and appreciation, and therefore to augment the chances for clinical and research co-operations in the future.

Enjoy the paediatric oncology contributions in MEMO. Your feedback is welcome and will help the editors to improve the journal as well as to reach the aims for which we set forth on this journey into paediatric oncology.
\end{abstract}

Michael N. Dworzak (Section Editor Paediatric Oncology) 